\title{
Molecular genetics of central nervous system tumors
}

\author{
Michael D. Taylor, M.D., Ph.D. \\ Division of Neurosurgery, Hospital for Sick Children, University of Toronto, Ontario, Canada
}

Neurosurgeons are often the gatekeepers of the neurooncology world; most patients with a brain tumor will have their first therapeutic encounter with a neurosurgeon. In recent years, molecular biology and molecular genetics have become part of the day-to-day management in a small but significant minority of patients with brain tumors by providing tools for the classification and prognostication of various neoplasms. With an ever more informed and demanding patient population that has access to the Internet, neurosurgeons who treat patients with brain tumors need to be aware of some of the issues raised by molecular biology and molecular genetics. Indeed, new papers on brain tumor biology are being published every day, and the amount of research that has successfully traveled from the bench to the bedside is accruing. Neurosurgeons should be proud that they have played a large role in generating a great deal of the data currently known about primary brain tumors.

In this issue of Neurosurgical Focus we have included a number of articles on specific types of brain tumors (that is, oligodendrogliomas), hereditary syndromes that predispose to brain tumors (that is, neurofibromatosis Type 2 ), and some of the current techniques used in neurooncology research (that is, modern cytogenetics). These articles nicely summarize the current state of knowledge in neurooncology for their given topics, and attempt to review many of the clinically relevant findings from neurooncology laboratories across the world. These articles should help to inform surgeons who treat tumors of the central nervous system about issues relevant to the care of their patients as well as serving as an interesting review for members of the neurooncology community. 